EPJ Web of Conferences 61, 06002 (2013)

DOI: $10.1051 /$ epjconf/ 20136106002

(C) Owned by the authors, published by EDP Sciences, 2013

\title{
Parsec-Scale Kinematic and Polarization Properties of MOJAVE AGN Jets
}

\author{
Matthew L. Lister ${ }^{1, a}$ for the MOJAVE collaboration ${ }^{b}$ \\ ${ }^{1}$ Department of Physics, Purdue University, 525 Northwestern Avenue, West Lafayette, IN 47907, USA
}

\begin{abstract}
We describe the parsec-scale kinematics and statistical polarization properties of 200 AGN jets based on $15 \mathrm{GHz}$ VLBA data obtained between 1994 Aug 31 and 2011 May 1. Nearly all of the 60 most heavily observed jets show significant changes in their innermost position angle over a 12 to 16 year interval, ranging from $10^{\circ}$ to $150^{\circ}$ on the sky, corresponding to intrinsic variations of $\sim 0.5^{\circ}$ to $\sim 2^{\circ}$. The BL Lac jets show smaller variations than quasars. Roughly half of the heavily observed jets show systematic position angle trends with time, and 20 show indications of oscillatory behavior. The time spans of the data sets are too short compared to the fitted periods ( 5 to $12 \mathrm{y}$ ), however, to reliably establish periodicity. The rapid changes and large jumps in position angle seen in many cases suggest that the superluminal AGN jet features occupy only a portion of the entire jet cross section, and may be energized portions of thin instability structures within the jet. We have derived vector proper motions for 887 moving features in 200 jets having at least five VLBA epochs. For 557 well-sampled features, there are sufficient data to additionally study possible accelerations. The moving features are generally non-ballistic, with $70 \%$ of the well-sampled features showing either significant accelerations or non-radial motions. Inward motions are rare $(2 \%$ of all features), are slow $(<0.1$ mas per y), are more prevalent in BL Lac jets, and are typically found within 1 mas of the unresolved core feature. There is a general trend of increasing apparent speed with distance down the jet for both radio galaxies and BL Lac objects. In most jets, the speeds of the features cluster around a characteristic value, yet there is a considerable dispersion in the distribution. Orientation variations within the jet cannot fully account for the dispersion, implying that the features have a range of Lorentz factor and/or pattern speed. Very slow pattern speed features are rare, comprising only $4 \%$ of the sample, and are more prevalent in radio galaxy and BL Lac jets. We confirm a previously reported upper envelope to the distribution of speed versus beamed luminosity for moving jet features. Below $10^{26} \mathrm{~W} \mathrm{~Hz}^{-1}$ there is a fall-off in maximum speed with decreasing $15 \mathrm{GHz}$ radio luminosity. A preliminary analysis of the multi-epoch jet polarization properties indicates a wide range of behavior in the core electric vector position angles over time, with the latter remaining relatively stable in some jets, and varying rapidly in others. The fractional polarization level generally increases down the jet, and high-synchrotron peaked (HSP) blazars tend to have lower core fractional polarization levels. A general trend of decreasing maximum jet speed for higher synchrotron peaked blazars further suggests lower Doppler factors in the radio-emitting jets of HSP BL Lac objects.
\end{abstract}

\section{Introduction}

High resolution multi-epoch radio observations of jetted outflows associated with active galactic nuclei (AGN) have contributed substantially to our understanding of the immediate environments of supermassive black holes, by providing direct measurements of jet flow kinematics and magnetic field properties. The VLBA $2 \mathrm{~cm}$ Survey [1] sampled the jet kinematics of 110 AGN, and was succeeded in 2002 by the MOJAVE program, which added full polarization imaging and defined a complete northernsky radio flux density-limited sample [2]. Kinematic results for 127 MOJAVE jets based on data spanning 19942007 were presented by [3] and [4]. They showed that bright jet features typically exhibit apparent superluminal speeds and accelerated motions. These findings are consis-

\footnotetext{
ae-mail: mlister@purdue.edu

$\mathrm{b}_{\text {www.physics.purdue.edu/MOJAVE/team.html }}$
}

tent with the widely accepted picture of high bulk Lorentz factor jets viewed at angles very close to the line of sight.

The MOJAVE program has confirmed an important trend, first reported by [5] in the Caltech-Jodrell AGN survey, in which jets with the fastest superluminal speeds all tend to have high Doppler boosted radio luminosities. To first order, such a trend might be expected from orientation and Doppler boosting effects, but an analysis by [6] and Monte Carlo simulations presented in [3] indicated that there is a correlation between intrinsic jet speed and intrinsic (de-beamed) luminosity present in the population. In the absence of such a correlation, we would expect to see highly superluminal jets at much lower boosted radio luminosities.

In order to further investigate these issues, we expanded the MOJAVE program in 2006 to include regular VLBA imaging of additional low-luminosity AGN jets. In 
2009, we expanded the sample again to encompass new $\gamma$-ray loud blazar jets detected by Fermi [7].

We have recently published new VLBA $15 \mathrm{GHz}$ images of the original 135 source MOJAVE flux-density limited sample obtained between 2007 and 2011, as well as VLBA images of of 124 additional AGN from three new AGN jet samples [8] . Here we present a kinematic analysis of these and prior data dating back to 1994, as well as preliminary results from our ongoing analysis of the multiepoch VLBA polarization images. The full set of images and data tables can be found in [8]. We adopt a cosmology with $\Omega_{m}=0.27, \Omega_{\Lambda}=0.73$ and $H_{o}=71 \mathrm{~km} \mathrm{~s}^{-1} \mathrm{Mpc}^{-1}$ throughout.

\section{MOJAVE AGN Samples}

The data presented here come from three MOJAVE AGN samples in the sky region $\delta>-30^{\circ}$ with selection criteria as follows:

Radio-selected MOJAVE 1.5 Jy sample: This includes all known AGN with $15 \mathrm{GHz}$ VLBA flux density exceeding $1.5 \mathrm{Jy}$ at any epoch during the period 1994.02010.0. There are 183 AGN in total, with the sample being heavily dominated by flat spectrum radio quasars (78\%) and BL Lac objects (16\%). The optical classifications are $98 \%$ complete, with redshifts available for $96 \%$ of the sample.

$\gamma$-ray-selected 1FM sample: For the joint LAT teamMOJAVE study of [7], we constructed a $\gamma$-ray sample based on the 11-month First Fermi AGN catalog [9] with average integrated $>0.1 \mathrm{GeV}$ energy flux $\geq 3 \times$ $10^{-11} \mathrm{erg} \mathrm{cm}^{-2} \mathrm{~s}^{-2}$ between 2008 August 4 and 2009 July 5 and galactic latitude $|b|>10^{\circ}$. The sample is complete with respect to $\gamma$-ray flux, with the exception of two $\gamma$-ray sources that were dropped since they had no unambiguous radio counterpart. There are $116 \mathrm{AGN}$ in total, 56 of which are in common with the MOJAVE $1.5 \mathrm{Jy}$ sample. Like our radio-selected sample, it is heavily dominated by blazars, but contains a larger fraction of BL Lac objects $(36 \%)$. The remainder of the sample are quasars, with the exception of the nearby radio galaxy NGC 1275 (3C 84) and the narrow-line Seyfert 1 galaxy PMN J0948+0022.

Low-luminosity compact AGN sample: In 2006 we expanded the MOJAVE program to include regular VLBA observations of 16 AGN with VLBA $15 \mathrm{GHz}$ luminosities below $<10^{26} \mathrm{~W} \mathrm{~Hz}^{-1}$. These were chosen from the VLBA Calibrator Survey [10-15], based on the following criteria: i) $8 \mathrm{GHz}$ VLBA flux density greater than $0.35 \mathrm{Jy}$, ii) $z \leq 0.3$. By adding the AGN already in the MOJAVE program which met these criteria, we obtained a final sample of 43 low-luminosity compact AGN.

These three samples, comprising 259 AGN in total, provide a broad cross-section of AGN types among bright, compact radio sources. Since only 200 of these sources had at least 5 VLBA epochs as of May 2011, the kinematic data are incomplete. In particular, the AGNs with missing data tend to be among the weaker radio and $\gamma$-ray selected AGNs that were added later in the MOJAVE survey. A statistical inter-comparison of these samples will be presented once a full, unbiased dataset has been collected.

\section{VLBA Observations and Data Analysis}

The $15 \mathrm{GHz}$ VLBA data used in this study were obtained either from the VLBA archive, the VLBA $2 \mathrm{~cm}$ Survey program, or the MOJAVE program. In the latter, AGNs were monitored at intervals ranging from every 3 weeks to every 3 years, depending on the angular expansion rate of the individual AGN jets. The data were reduced using standard methods as described in [16].

We modelled the $(u, v)$ visibility data at all AGN epochs using a series of Gaussian components in the Difmap software package [17]. In the majority of cases, we used circular Gaussians for jet features, and occasionally (when necessary) elliptical Gaussians for the core feature. The latter was typically the brightest feature at the extreme end of a one-sided jet in most sources. In some instances, it was not possible to robustly cross-identify the same components in a jet from one epoch to the next. After discarding these, 887 robust components with at least 5 data epochs were available for more detailed kinematics analysis.

We performed two sets of kinematics analyses on the robust Gaussian jet components in our sample. The first assumed a simple non-accelerating, two-dimensional vector fit to the component position over time, referenced to the core component (which we presumed to be stationary). For the components which had measurements at 10 or more epochs, we also performed a constant acceleration fit (as described in [3], which yielded kinematic quantities at a reference (middle) epoch. If these showed a significant $(\geq 3 \sigma)$ acceleration, we adopted the kinematic values from the acceleration fit.

\section{Discussion}

\subsection{Jet Kinematics Analysis}

We examined each component with significant proper motion $\left(\mu \geq 3 \sigma_{\mu}\right)$ for non-radial motion by comparing the mean position angle of the component $\langle\vartheta\rangle$ with its proper motion vector direction $\phi$. We flagged any component for which the angular offset $|\langle\vartheta\rangle-\phi|$ was $\geq 3 \sigma$ from either $0^{\circ}$ or $180^{\circ}$ as "non-radial", and "inward" if the offset was significantly greater than $90^{\circ}$. We made these determinations using the $|\langle\vartheta\rangle-\phi|$ values from the acceleration fit for significantly accelerating components, or from the vector fits otherwise. Of the $739(\geq 3 \sigma)$ motion components classified as robust, $282(38 \%)$ exhibit significant non-radial motion.

We find 16 statistically significant inward component motions in 10 different jets (6 BL Lac objects, 2 quasars, and 2 radio galaxies). BL Lac jets are statistically overrepresented in this group, given that they make up only $20 \%$ of the 200 AGN jets we analyzed for kinematics. We do not find anything otherwise distinctive about these particular BL Lacs, and the small number statistics make it impossible to draw any firm conclusions as to the cause of this overrepresentation. We also note that 4 of the 10 inward component motion jets are BL Lacs with no measured redshift. The latter is not unexpected, however, since 
Table 1. Summary of Kinematics Analysis Results

\begin{tabular}{lr}
\hline Property & Number \\
\hline AGN analyzed for kinematics & 200 \\
Jet components classified as robust & 887 \\
Robust slow pattern speed components & $38(4 \%)$ \\
Robust jet components with $(\geq 3 \sigma)$ measured speeds & $739(83 \%)$ \\
Robust inward-moving $(\geq 3 \sigma$ speed) components & $17(2 \%)$ \\
Components with significant $(\geq 3 \sigma)$ non-radial motion & $282(38 \%)$ \\
Components analyzed for acceleration & $547(62 \%)$ \\
Components with significant acceleration & $212(39 \%)$ \\
Components with significant perpendicular acceleration & $99(18 \%)$ \\
Components with significant parallel acceleration & $155(28 \%)$ \\
\hline
\end{tabular}

by definition BL Lacs have weak emission lines or featureless optical spectra that often make it difficult or impossible to measure their redshifts.

The inward motions are all typically slow, with a median value of $33 \mu \mathrm{as}^{-1}$, and none are faster than $100 \mu$ as $\mathrm{y}^{-1}$. Considering only the AGN with a known redshift, the inward components of $1458+718$ are the only ones which appear significantly superluminal, ranging from $1.4 \mathrm{c}$ to $4.6 \mathrm{c}$. With the exception of $1458+718$, $2021+614$, and $2230+114$, the inward motions all occur within $\sim 1$ mas of the core, in typically the innermost component. In particular, the innermost two jet components of two TeV-emitting BL Lacs in our sample: 0219+428 (3C 66A) and 1219+285 (W Comae) are both inward-moving. The small velocities and core separations of these moving components may indicate that the core is not a stable reference point in these two jets. We did not find any significant inward motions in the other 15 currently known TeV-emitting AGN jets which we analyzed.

There are many scenarios under which apparent inward motions can be produced, including: i) curved jet motions which cross the line of sight, ii) non-stationarity of the apparent core feature, due to one or more newly emerging features below the interferometric resolution level, iii) a misidentification of the true stationary core with a moving feature, iv) internal brightness changes in a large, diffuse jet feature, and v) apparent backward pattern speeds not associated with the flow. The very small numbers of inward-moving features strongly rules out a fully random pattern speed scenario for the motions, in which equal numbers of inward and outward motions would be expected.

\subsection{Parsec-Scale Jet Orientation Variations}

The tendency for the parsec-scale jets of blazars to change their position angles on the sky with time has been solidly established via long term VLBI studies of several individual AGN (see [18] for a recent review). The exact origin of the wobbling is not clear, although accretion disk precession, orbital motion of the accretion system, or instabilities in the jet flow have all been suggested. A main signature of precession is sinusoidal variations in the jet position angle, and evidence for this has been seen in blazars such as 3C 273 [19], 3C 345 [20], 0716+714 [21], BL Lac [22], and M81 [23]. Other jets have displayed monotonic position angle swings with no evidence of periodicity (e.g., 3C 279: [24]; NRAO 150: [25]).
Until now, there has been no systematic survey of jet position angle variations in a large blazar sample. Using our extensive MOJAVE database, we analyzed the innermost jet regions of 60 AGN from our radio-selected sample for which we have obtained 20 or more VLBA epochs over a minimum twelve year period. We excluded several well-monitored jets in our sample for reasons of core identification uncertainty, counterjet emission, or highly curved jet structure within one mas of the core. We determined the innermost jet position angle at each epoch by taking a flux density-weighted position angle average of all clean components above 3 times the image noise level in the annular region from 0.15 mas to 1 mas from the core. We also explored other methods, such as using the position angle of the innermost Gaussian modelfit component, or multiple components within a particular distance from the core, but the derived position angles were more influenced by choices made for the fitted Gaussians (e.g., elliptical versus circular, and total number of Gaussians in the inner jet region). Our position angle measurement method is subject to errors associated with wandering of the core component position due to changes in opacity or the emergence of new components below our resolution level. These are likely small, however, since they would create correlated apparent motions in components located downstream, which we have not detected in our data.

We find that the innermost jet position angles vary considerably over the 12 to 16 year intervals covered by our data, with ranges up to $150^{\circ}$ in some jets. The typical circular standard deviation in position angle is $\sim 10^{\circ}$. The quasars and BL Lac objects differ significantly in their ranges, according to Kolmogorov-Smirnov tests on their range $\left(p_{\text {null }}=1.1 \%\right)$ and standard deviation $\left(p_{\text {null }}=1.3 \%\right)$ distributions. It is unclear whether the smaller overall variations we see in the BL Lac innermost jet position angles is an intrinsic effect, or because they are oriented at slightly larger angles to the line of sight than quasars.

Some jets (e.g., NRAO $150=0355+508)$ show a very wide range of inner jet position angle and Gaussian component position angles, likely because the viewing angle to the inner jet lies within the opening angle of the (presumably conical) outflow. In other cases, such as 3C 273 $(1226+023)$, the jet is tranversely resolved into multiple features, some of which are moving along different position angles at nearly the same radial distance from the core. Since stacked-epoch VLBA images [16] often show a smooth conical jet intensity profile in highly variable ejection angle jets such as $1308+326$, the simplest interpretation is that individual emerging features do not fill the entire cross-section of the flow. Instead, features are ejected within a finite width "ejection cone", which becomes apparent only in a stacked-epoch image. The apparent opening angle of this cone is exaggerated by projection effects by a factor of $\sin \theta$. Thus for typical blazar jet viewing angles of $\theta \lesssim 5^{\circ}$, the intrinsic ejection cone full opening angle is likely $\sim 0.5^{\circ}$ to $\sim 2^{\circ}$, based on the position angle range distribution in our sample. AGN jets can therefore appear "bent" in a single-epoch, limited dynamic range VLBI image, whereas in reality what is vis- 

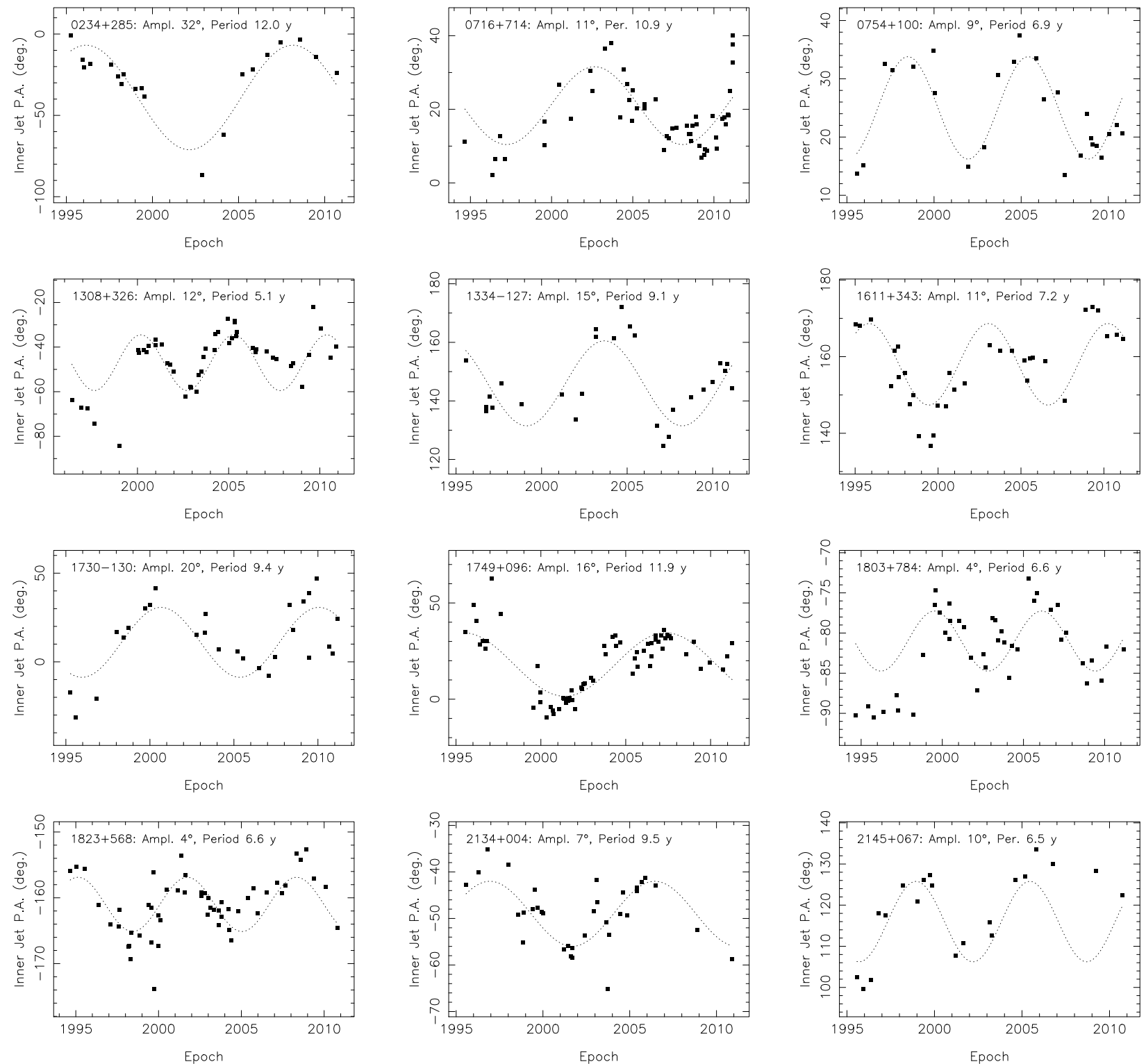

Figure 1. Innermost jet position angle versus time plots for 12 selected MOJAVE jets displaying oscillatory trends. The dotted lines represent the best sine curve fits to the data, based on the peak period in the Lomb-Scargle periodogram.

ible is just the portion of the jet that is currently experiencing enhanced synchrotron emission, due to the passage of several very bright features. These features may represent flow instabilities driven at the nozzle (e.g., [26]), which vary within the jet over time and in turn influence the kinematics downstream.

The individual AGNs in our sample show a variety of inner jet evolution patterns, which we have classified into four general categories. There are 14 jets which display a monotonic trend of inner jet position angle with time, 5 which show a back and forth trend, 12 which show more than one cycle of back and forth motion (oscillatory), and 29 with no discernible trend. Within these categories, 11 jets exhibited one or more abrupt jumps in position angle, caused by a new feature emerging from the core with a significantly different trajectory than previously ejected features.
We performed linear regression fits on all of the jets showing monotonic trends (in some cases, we had to omit segments of the data from the fit due to abrupt jumps in position angle). The most rapidly varying jet, at $9.8 \pm 1^{\circ} \mathrm{y}^{-1}$, is $0355+508$ (NRAO 150). Our measurement is consistent with the swings of up to $11^{\circ} \mathrm{y}^{-1}$ seen previously in this jet by [25] in VLBA images at 43 and $86 \mathrm{GHz}$. The other monotonically changing jets in our sample show swings of typically a few degrees per year.

We looked for evidence of periodicity in all 60 jets using Lomb-Scargle periodograms, which are well-suited for unequally sampled time-series data [27, 28]. With the exception of $0234+285$ and $2145+067$, the 12 jets shown in Figure 1 have significant $(\geq 2 \sigma)$ peaks at the periods indicated on each sub-panel, which range from 5 to 12 y. The dashed lines represent the best sinusoid fits to the data for the indicated Lomb-Scargle period. For these fits we allowed the amplitude, mean, and phase to vary in or- 

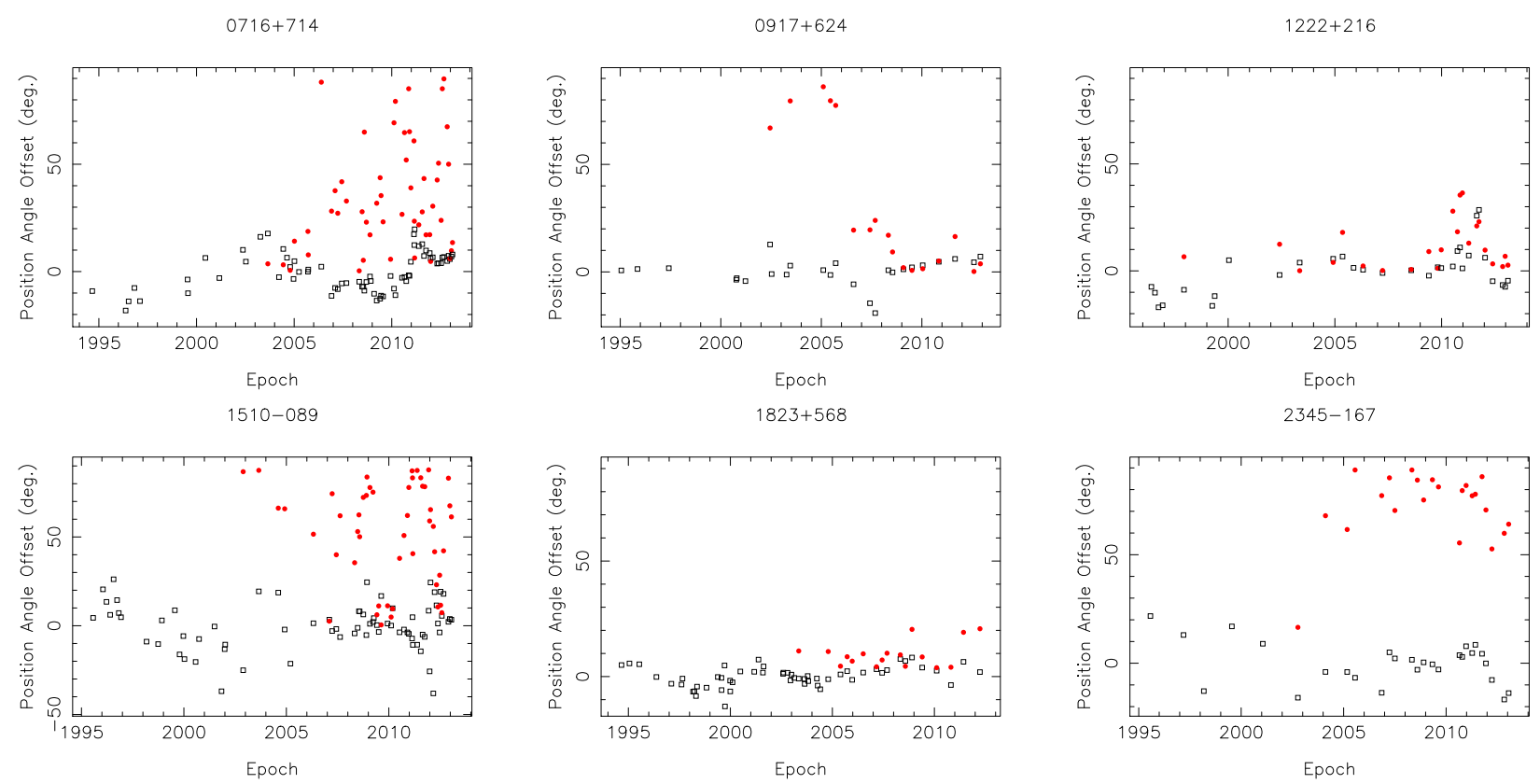

Figure 2. Plots of innermost jet position angle (black unfilled squares) and core electric vector position angle offset (red filled circles) versus time for selected MOJAVE jets showing a range of behaviors. The jet position angles are rotated such that the median value is at zero degrees.

der to find the best absolute $\chi^{2}$ value. Assuming a typical Gaussian normal error of $2^{\circ}$ for the position angle measurements (based on the linear regression fit residuals to the monotonic trend sources), the best reduced- $\chi^{2}$ fit values are 1.5 for $0716+714$, and 2.5 for $1823+568$. We cannot reliably establish periodicity in any jet, however, due the lack of sufficient cycles in the data, and the fact that many of the fits have significant residuals (e.g., $1308+326,1803+784,2145+067)$, suggesting that the behavior is more complex than a single sinusoidal variation.

Using the MOJAVE VLBA polarization data, we have begun an analysis of the electric vector position angle (EVPA) of the core features, to compare these with the inner jet position angle over time. Our preliminary results indicate a wide range of behavior, with some jets maintaining EVPA orientations parallel to, or perpendicular to the jet for long periods, while others show constantly variable EVPAs (Fig. 2). Although Faraday rotation of the core EVPAs is minimal at $15 \mathrm{GHz}\left(\sim 10-15^{\circ}\right.$; [29]), some high rotation measures are occasionally seen. A more complete statistical analysis of the core EVPA properties, including the behavior during superluminal component ejections, will presented in a forthcoming paper.

\subsection{Dispersion of Apparent Speeds}

A longstanding question in AGN jet kinematics is whether the bright features in a given jet all tend to propagate at a characteristic speed which represents the true flow. In [3] we found that roughly $20 \%$ of the jets had one or more features which moved significantly slower than the other features in the jet. These slow pattern speed features could be the result of either stationary shocks in the flow, or jet bending across the line of sight. We have repeated this analysis for the current data set, using more stringent criteria.

We first tabulated a maximum speed for each jet by considering the component with the fastest $\geq 3 \sigma$ speed. If no component in the jet had $\mathrm{a} \geq 3 \sigma$ speed, we lowered the criterion to $>2 \sigma$. In the case of $0355+508,1329-049$, and $1520+319$, which had no $\geq 2 \sigma$ components, we used the component speed with the smallest measured error. We dropped 16 jets from the analysis since they had no robust components with which to measure a maximum speed. We flagged components as "Slow Pattern Speed" (SPS) if they had i) no statistically significant acceleration, ii) a speed less than $20 \mu \mathrm{as}^{-1}$, and iii) a speed at least 10 times slower than the fastest component in the jet. We found 38 such components in 29 different jets (14 quasars, 10 BL Lacs, and 4 radio galaxies).

A significantly higher fraction of BL Lacs and radio galaxies in our sample contain SPS components $(\sim 25 \%)$ as compared to quasar jets $(10 \%)$. We detect no significant differences in the redshift distributions of SPS and non-SPS jets, but their median VLBA $15 \mathrm{GHz}$ luminosity distributions are different at the $98 \%$ confidence level according to a Kolmogorov-Smirnov test. Of the 37 jets with radio luminosity above $10^{28} \mathrm{~W} / \mathrm{Hz}$, only one has a SPS component (the quasar 2134+004). This is consistent with numerical simulations (e.g., [30, 31]) which show that more highly relativistic jets exhibit fewer compact internal structures and less overall instability.

In order to examine the dispersion of speeds within individual jets in more detail, we calculated a normalized statistic $D=\left(\mu_{\max }-\mu_{\min }\right) /\left(\mu_{\max }+\mu_{\min }\right)$ for all 75 jets which had at least five robust components, as well as a 


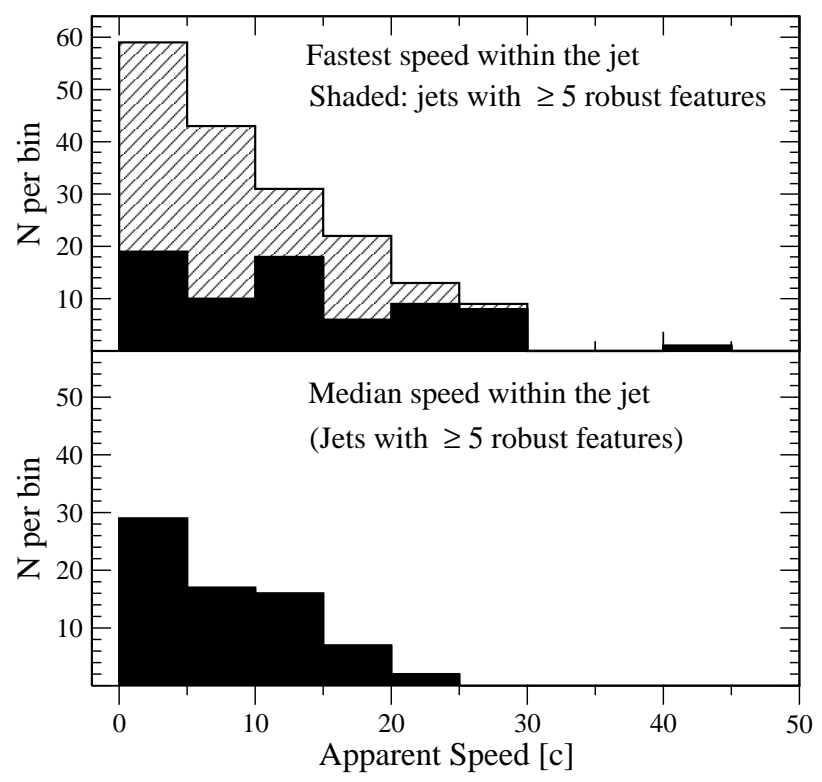

Figure 3. Distributions of fastest and median speed for jets in our sample with measured redshifts. The shaded histograms are for jets having at least 5 robust components.

median speed and rms dispersion value. Over half of these jets have $D$ values above 0.8 , indicating that in most cases, the overall range of apparent speed within a jet is comparable to its maximum speed. Removing the SPS components from the analysis did not change this general result.

Although we find there can be a large range of apparent speed within a jet, the speeds usually cluster around a median value, indicating they are not random. The median rms dispersion of speed within a jet is $2.8 c$. This is significantly less than the overall dispersion of the median values $(6.7 c)$. Furthermore, the distributions of maximum and median speed in the sample are not uniformly distributed in either angular or spatial measure, but are instead peaked at low values (Fig. 3).

In Figure 4 we show the binned distribution of the speed dispersion statistic $D=\left(\mu-\mu_{\text {median }}\right) / \mu_{\text {median }}$, the fractional difference with respect to the median speed in the jet. The distribution is nearly normal, although with significant kurtosis (more sharply peaked than Gaussian, according to an Anscombe-Glynn test). There is no significant skewness if the outlier at $2.8(1823+568)$ is omitted (D'Agostino test). One might expect an asymmetric distribution (positively skewed) if some fraction of the features travel at the true flow speed and none of them exceed it, but we do not see evidence of this in the data. Since we can typically identify more robust components in the jets for which we have the longest monitoring intervals, we might also expect to see a positive trend between the maximum speed of a jet and the number of robust components, but this is not evident in our data. Our findings thus suggest that in general, high ejection rate blazar jets tend to eject features with apparent speeds which cluster about a speed that is characteristic to each individual jet.

A dispersion of apparent speeds within a jet might be expected in the context of our model presented in Sec-

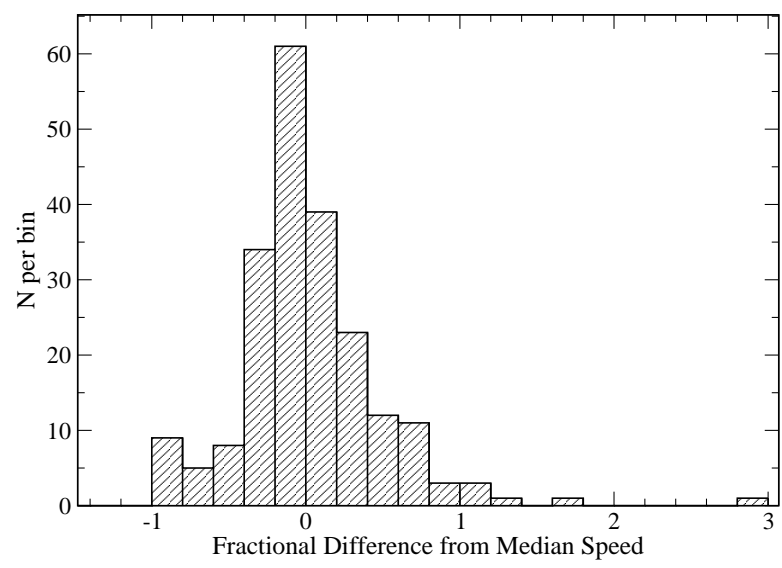

Figure 4. Overall normalized speed distribution of moving features within jets having at least 10 robust components. The fractional difference is defined as $(\mu-$ $\left.\mu_{\text {median }}\right) / \mu_{\text {median }}$.

tion 4.2, in which features do not fill the entire jet cross section, and emerge along a radial streamline within a conical outflow. Evidence of thin ribbon-like instabilities has been seen in the transversely resolved jet 3C 273 [32] and the quasar S5 0836+710 [33]. The latter authors conclude that the ridgeline in VLBI images may correspond to a helically twisted pressure maximum within the jet, which slowly varies in position on decadal timescales. In the case of 3C 273, polarimetric VLBA rotation measure maps made at different epochs by [34], [35], and [29] indicate that different parts of the jet are being illuminated at different times. Similar behavior has also been reported in the broad line radio galaxy 3C 120 by [36].

In our proposed scenario we would expect a range of apparent viewing angle roughly equal to the full opening angle of the ejection cone, and a corresponding range in apparent speed. In [8] we examined the range of expected apparent speed for conical jets with a fixed $2^{\circ}$ full opening angle versus on-axis viewing angle, and concluded that the spread in apparent speed we see in individual jets cannot be wholly attributed to a spread in streamline viewing angle. The moving features must therefore also have an intrinsic range of bulk Lorentz factor and/or pattern speed.

\subsection{Trends With Apparent Speed}

\subsubsection{Speed versus distance down the jet}

In our analysis of MOJAVE and VLBA archive images from 1994-2007 ([3],[4]) we found that close to the base of the jet, features tended to show increasing rather than decreasing apparent speed. This suggests that AGN jet flows are still being organized on pc-scales, a favored possible site for high-energy photon production. In order to test for this trend in our current dataset, we have plotted the apparent speed of all robust components versus their mean projected distance from the core in Figure 5. We have omitted components with non-radial or inward motions, and have included lines of constant age which assume steady radial motion over the indicated time period. Since the components in our sample have a wide range 
of ages, this would tend to suppress any artificial trend of higher mean distance for faster components. However, no components are found in the upper left corner of the plot, since these fast components will have higher mean core distance values and quickly evolve towards the right hand side of the diagram. Also, sufficient time must pass to gather sufficient epochs for an apparent speed measurement in our survey. Components are also absent from the lower right "graveyard" region since these evolved components have likely undergone considerable adiabatic expansion and synchrotron energy losses, thereby dropping their flux densities below the threshold for which we can robustly measure their centroids and speeds. There is also a notable deficit of components above $\sim 0.4 \mathrm{c}$ within $\sim 1$ $\mathrm{pc}$ of the core, which would be even more pronounced without the inclusion of the numerous components associated with the two-sided jets of the gigahertz-peaked spectrum galaxy NGC 1052. The two broad line radio galaxies 3C 111 and 3C 120 also occupy a distinct region among the radio galaxy data plotted in Figure 5.

The overall distribution of the components in the plot indicates a positive correlation of speed with core distance for radio galaxies and BL Lac objects, even after partialling out redshift. This trend needs to be confirmed, however, using a larger, complete AGN sample which extends below our low-luminosity sample limit of $0.35 \mathrm{Jy}$. It is not possible to assess the existence of a trend for the quasars due to the observational selection effects described above. Because of the low space density of bright quasars at low redshift and the fixed angular resolution limit of our survey, there is likely an artificial deficit of quasar jet components in the region below $1 \mathrm{c}$ and within $\sim 1 \mathrm{pc}$ of the core, precisely where data are needed to test for a possible trend.

\subsubsection{Speed versus luminosity}

A plot of apparent speed versus luminosity for the robust jet components (Fig. 6) reveals a distinct deficit of fast components at low luminosities. The apparent upper envelope to this distribution has been discussed in several studies ([2, 3, 5, 6, 37, 38]), and roughly matches the locus of points for a jet of fixed intrinsic luminosity and bulk Lorentz factor, oriented at different angles to the line of sight (for $L>10^{23} \mathrm{~W} \mathrm{~Hz}^{-1}$ ). The parametric red curve in Figure 6) is drawn for a jet with $\Gamma=35$, $L_{\text {int }}=10^{23} \mathrm{~W} \mathrm{~Hz}^{-1}$, and Doppler boost $\delta^{2.7}$. This curve is representative only, since jets in the population have a range of Lorentz factors and intrinsic luminosities, and thus a family of such curves exist, as described by [6]. The upper left and lower right regions of the plot (delimited by the dot-dashed lines) are not sampled by our survey. The deficit region located below the red curve and below $10^{23} \mathrm{~W} \mathrm{~Hz}^{-1}$ is partly due to the incompleteness and relatively high flux density cutoff of our low-luminosity sample. The overall fall-off in the upper edge of the distribution from $10^{26} \mathrm{~W} \mathrm{~Hz}^{-1}$ to $10^{23} \mathrm{~W} \mathrm{~Hz}^{-1}$ is not the result of survey bias or selection effects, however, and reflects both the existence of a maximum jet Lorentz factor in the parent population $(\sim 40)$, and an intrinsic correlation between flow speed and luminosity in AGN jets. The relatively sharp edge to the upper envelope in Figure 6, as well as the relatively unchanging distribution of apparent speed in luminosity bins above $10^{26} \mathrm{~W} \mathrm{~Hz}^{-1}$ imply that intrinsically powerful AGN jets have a wide range of Lorentz factors up to $\sim 40$, while intrinsically weak jets are only mildly relativistic.

\subsection{Accelerating Components}

Theoretical models of the acceleration and collimation of blazar jets indicate that the strong magnetic fields associated with the putative supermassive black hole/accretion disk system play a key role in the initial acceleration and collimation of the jet [e.g., 39]. While some models indicate that this process is largely complete with the conversion of Poynting flux to flow energy on sub-pc scales [e.g., 40], there may still be significant magnetic [41] or hydrodynamic acceleration which extends to parsec or decaparsec scales.

In our previous analysis of MOJAVE data from 19942007 [4], we found that accelerated motions with respect to the mean apparent velocity vector $\vec{\beta}_{\text {obs }}$ were common, with significant parallel accelerations seen in roughly one third of our sample, and significant perpendicular accelerations in about one fifth of our sample. Due to the limited number of available epochs, we were only able to analyze 311 of the 526 moving components for possible accelerated motion. Using our new data spanning up to 2011 May 1, we have performed the same analysis on 547 of 887 moving components which had at least 10 epochs. We summarize our overall acceleration results in Table 1. Our incidence rates of parallel accelerations (28\%) and perpendicular accelerations $(18 \%)$ are consistent with those reported in [4]. Nearly $40 \%$ of the components analyzed show significant acceleration of either type. A substantial number of components showed no significant acceleration, but had non-radial motion vectors. If we assume that these components accelerated some time prior to our monitoring interval, the fraction of moving components showing accelerations rises to $70 \%$.

\subsection{Polarization and other trends}

All of the MOJAVE VLBA observations since 2002 have been carried out in dual polarization mode, and first-epoch polarization results on the radio flux limited sample were published in [2] and [42]. Our analysis of the multi-epoch data is currently underway, and has confirmed several of our earlier results, including a general trend of increasing linear fractional polarization down the jet [2], and a tendency for the cores of high-synchrotron peaked (HSP) blazars to have lower fractional polarizations [7]. The latter class emits the bulk of its synchrotron radiation in the optical to UV region, and is primarily composed of $\mathrm{BL}$ Lac and TeV-detected AGNs [43]. Our preliminary results indicate a general trend of decreasing apparent jet speed 


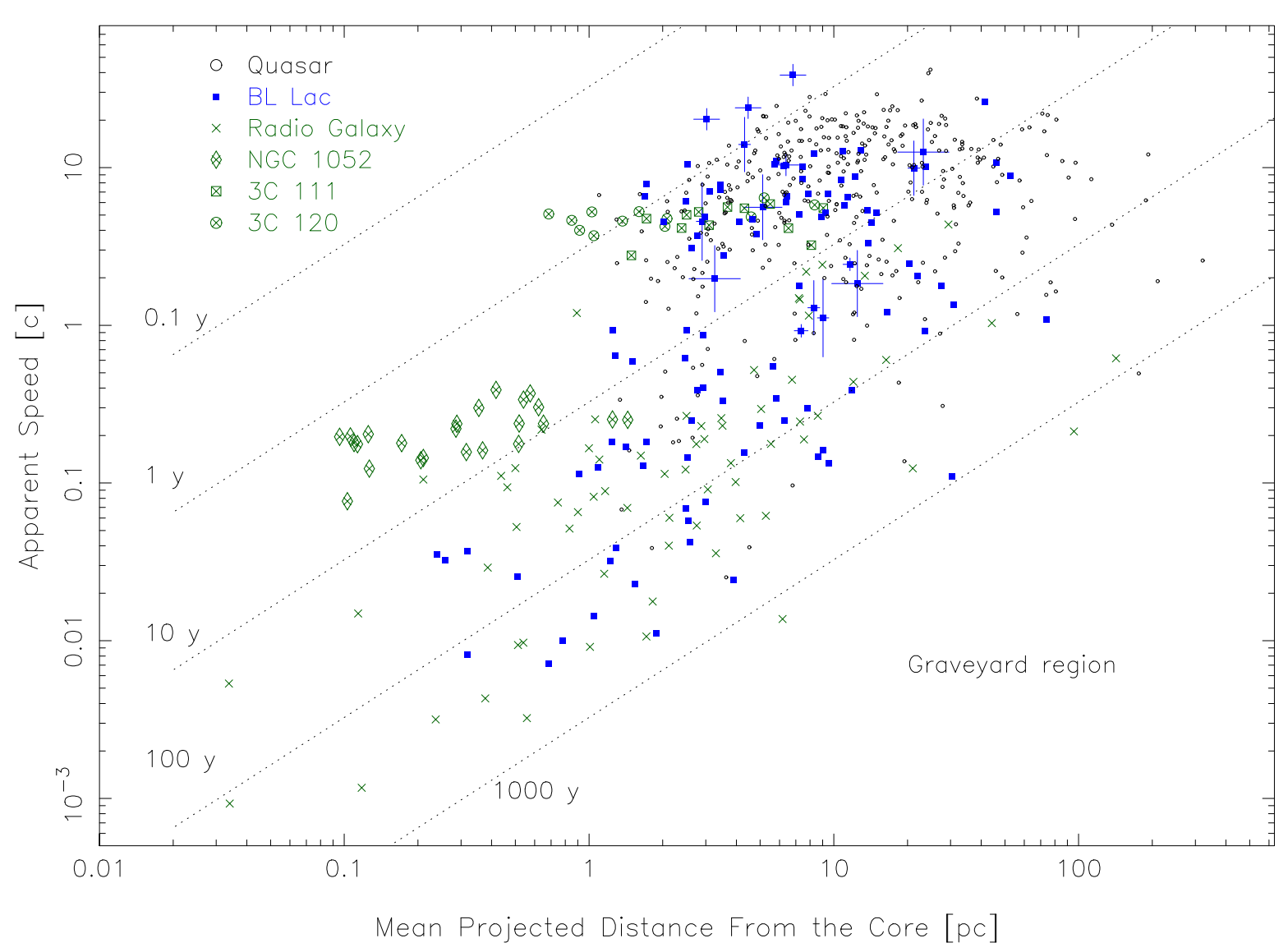

Figure 5. Apparent speed versus mean projected distance from the core in parsecs for all robust jet components (excluding non-radial and inward components). The green crosses denote components in radio galaxies, the black circles quasars, and the blue squares BL Lacs. The jet components of two broad-lined radio galaxies (3C 111 and 3C 120), as well as the gigahertz-peaked spectrum radio galaxy NGC 1052 are highlighted with distinct symbols. Error bars have been omitted for the purposes of clarity. For components in jets which do not have a spectroscopic redshift, we include range bars which correspond to their known redshift constraints. The dotted lines indicate lines of constant age for components that have moved steadily outward over the time period indicated. Newly emergent fast components rapidly evolve out of the top left region of the plot, while no components are found in the bottom right graveyard region since they will likely have faded well below the MOJAVE imaging sensitivity level.

versus synchrotron peak frequency (Fig. 7). This is consistent with other trends with core compactness and radio variability seen in our $\gamma$-ray selected 1FM sample [7] which suggest lower Doppler factors for the radio-emitting jets in HSP BL Lac objects. We are currently obtaining kinematic data on a much larger set of HSP jets to further investigate these trends.

\section{Summary}

We have analyzed the kinematics of 200 parsec-scale AGN jets based on VLBA observations spanning 1994 Aug 31 to 2011 May 1. These jets were drawn from a complete radio-selected AGN sample above $1.5 \mathrm{Jy}$, a complete $\gamma$-ray selected sample, and a representative low-luminosity radio jet sample. We obtained vector motion fits to 887 robust components which were positively cross-identified over at least 5 VLBA epochs. We also measured the acceleration properties of 557 components which had at least 10 VLBA epochs. Our main findings are as follows:

1. Nearly all of the 60 most heavily observed jets show significant changes in their innermost position angle $\left(20^{\circ}\right.$ to $150^{\circ}$ ) over a 12 to 16 year monitoring interval, with $\mathrm{BL}$ Lac jets showing smaller variations than quasars. The observed range corresponds to intrinsic (de-projected) varia-

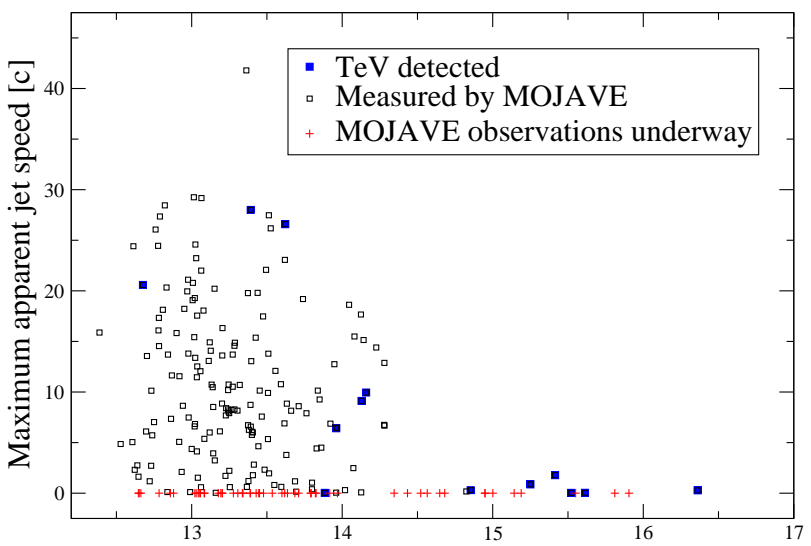

$\log$ rest frame synchrotron peak frequency $[\mathrm{Hz}]$

Figure 7. Plot of maximum measured jet speed versus synchrotron peak frequency for MOJAVE AGNs.

tions of $\sim 0.5^{\circ}$ to $\sim 2^{\circ}$. Roughly half of the heavily observed jets display a trend of innermost jet position angle with time. In the case of 12 jets, there is some evidence of oscillatory behavior, but the fitted periods ( 5 to $12 \mathrm{y}$ ) are 


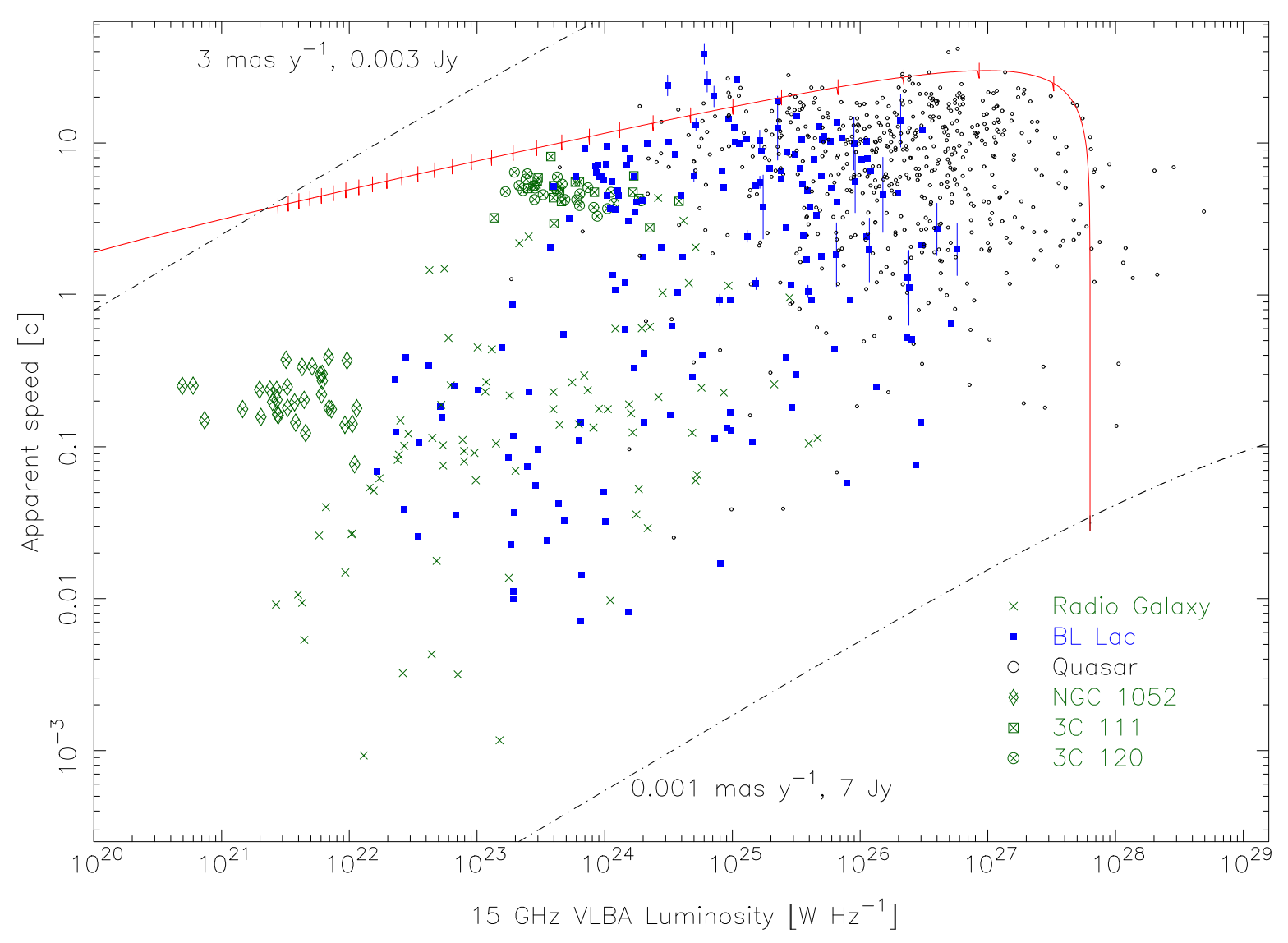

Figure 6. Apparent speed versus $15 \mathrm{GHz}$ luminosity for all robust jet components (excluding inward components). The green crosses denote components in radio galaxies, the black circles quasars, and the blue squares BL Lacs. Error bars have been omitted for purposes of clarity. For components in jets which do not have a spectroscopic redshift, we include vertical range bars which correspond to their known redshift constraints. The red curve shows the locus of possible locations for a jet component having intrinsic luminosity $10^{23} \mathrm{~W} \mathrm{~Hz}^{-1}$, Doppler boost $\delta^{2.7}$ and a Lorentz factor $\Gamma=35$, viewed at different angles to the line of sight. The tick marks are drawn at one degree intervals, ranging from a viewing angle of $30^{\circ}$ on the left to $1^{\circ}$ on the right. The dot-dashed lines correspond to observational limits; the regions in the top left and bottom right corners of the plot are not sampled by the survey.

too long compared to the length of the dataset to firmly establish periodicity. These periods are very short compared to expected precession timescales from warped accretion disk - black hole interactions associated with the Bardeen-Petterson effect [44]. Rather, we favor a conical jet model in which emerging features do not fill the entire cross-section of the flow. What is typically visible in a single-epoch, limited dynamic range VLBA image as a series of bright features may actually be the lit up portions of thin ribbon-like structures embedded within a broader conical outflow. These portions have been energized by a passing planar disturbance which originated in the VLBI core. As discussed by [33], the ribbon structures may arise from helical pressure maxima within the jet, and slowly vary in position on decadal timescales.

2. We examined the distribution of speeds within 75 jets which had at least 5 robust components. Within a particular jet, the speeds of the features usually cluster around a characteristic value, however, the range of apparent speed among the components is comparable to the maximum speed measured within the jet. This is not due uniquely to unusually slow pattern speed components (defined as having a speed less than 20 mas $^{-1}$ and at least 10 times slower than the fastest component in the jet), which comprise only $4 \%$ of all the components studied and are less prevalent in quasar jets. It is also too large to be solely due to differences in ejection angle. We conclude that the dispersion is at least partially due to an intrinsic distribution of bulk Lorentz factor and/or pattern speed.

3. Apparent inward motions are rare, with only $2 \%$ of the components having apparent velocity vectors greater than $90^{\circ}$ from the outward jet direction. They occur more frequently in BL Lac jets. All of the detected inward motions are slow $\left(\lesssim 0.1\right.$ mas $\left.^{-1}\right)$, and nearly all occur within 1 mas of the core component.

4. We find a trend of increasing apparent speed with distance down the jet for radio galaxies and BL Lac objects. The existence of a trend could not be evaluated for quasars due to unavoidable observational selection biases, as described in Section 4.4.1.

5. Accelerations are very common among the moving jet components. Of the 739 components with statistically significant $(\geq 3 \sigma)$ speeds, $38 \%$ exhibited significant nonradial motion, implying non-ballistic trajectories. We analyzed 547 components with at least 10 epochs, and found $28 \%$ to have significant accelerations parallel to the velocity vector, and $18 \%$ to have significant perpendicular accelerations. Nearly $40 \%$ showed significant acceleration of either type, and an additional $30 \%$ had non-radial motion vectors. 
6. A preliminary analysis of the multi-epoch jet polarization properties indicates a wide range of behavior in the core electric vector position angles over time, with the EVPA remaining relatively stable in some jets, and varying rapidly in others. The fractional polarization level generally increases down the jet, and high-synchrotron peaked (HSP) blazars tend to have lower core fractional polarization levels. A general trend of decreasing maximum jet speed for higher synchrotron peaked blazars suggests lower Doppler factors in the radio-emitting jets of HSP BL Lac objects.

\section{Acknowledgments}

The MOJAVE project was supported under NASAFermi grants NNX08AV67G and 11-Fermi11-0019. E. Ros was partially supported by the Spanish MINECO projects AYA2009-13036-C02-02 and AYA2012-38491C02-01 and by the Generalitat Valenciana project PROMETEO/2009/104, as well as by the COST MP0905 action 'Black Holes in a Violent Universe'. M. F. Aller and H. D. Aller were supported by NASA-Fermi GI grants NNX09AU16G, NNX10AP16G and NNX11AO13G and NSF grant AST-0607523. Y. Y. Kovalev was supported by the Russian Foundation for Basic Research (projects 12-02-33101, 13-02-12103), the basic research program "Active processes in galactic and extragalactic objects" of the Physical Sciences Division of the Russian Academy of Sciences, and the Dynasty Foundation. A. B. Pushkarev was supported by the "Non-stationary processes in the Universe" Program of the Presidium of the Russian Academy of Sciences. D. C. Homan was supported by NSF grant NSF AST-0707693. The National Radio Astronomy Observatory is a facility of the National Science Foundation operated under cooperative agreement by Associated Universities, Inc. This work made use of the Swinburne University of Technology software correlator [45], developed as part of the Australian Major National Research Facilities Programme and operated under licence.

\section{References}

[1] K.I. Kellermann, R.C. Vermeulen, J.A. Zensus, M.H. Cohen, AJ 115, 1295 (1998)

[2] M.L. Lister, D.C. Homan, AJ 130, 1389 (2005)

[3] M.L. Lister, M.H. Cohen, D.C. Homan, M. Kadler, K.I. Kellermann, Y.Y. Kovalev, E. Ros, T. Savolainen, J.A. Zensus, AJ 138, 1874 (2009)

[4] D.C. Homan, M. Kadler, K.I. Kellermann, Y.Y. Kovalev, M.L. Lister, E. Ros, T. Savolainen, J.A. Zensus, ApJ 706, 1253 (2009)

[5] R.C. Vermeulen, Proceedings of the National Academy of Science 92, 11385 (1995)

[6] M.H. Cohen, M.L. Lister, D.C. Homan, M. Kadler, K.I. Kellermann, Y.Y. Kovalev, R.C. Vermeulen, ApJ 658, 232 (2007)

[7] M.L. Lister, M. Aller, H. Aller, T. Hovatta, K.I. Kellermann, Y.Y. Kovalev, E.T. Meyer, A.B. Pushkarev, E. Ros, MOJAVE Collaboration et al., ApJ 742, 27 (2011)
[8] M.L. Lister, M.F. Aller, H.D. Aller, D.C. Homan, K.I. Kellermann, Y.Y. Kovalev, A.B. Pushkarev, J.L. Richards, E. Ros, T. Savolainen, ArXiv e-prints, 1308.2713 (2013)

[9] A.A. Abdo, M. Ackermann, M. Ajello, A. Allafort, E. Antolini, W.B. Atwood, M. Axelsson, L. Baldini, J. Ballet, G. Barbiellini et al., ApJ 715, 429 (2010)

[10] A.J. Beasley, D. Gordon, A.B. Peck, L. Petrov, D.S. MacMillan, E.B. Fomalont, C. Ma, ApJS 141, 13 (2002)

[11] E.B. Fomalont, L. Petrov, D.S. MacMillan, D. Gordon, C. Ma, AJ 126, 2562 (2003)

[12] L. Petrov, Y.Y. Kovalev, E. Fomalont, D. Gordon, AJ 129, 1163 (2005)

[13] L. Petrov, Y.Y. Kovalev, E.B. Fomalont, D. Gordon, AJ 131, 1872 (2006)

[14] Y.Y. Kovalev, L. Petrov, E.B. Fomalont, D. Gordon, AJ 133, 1236 (2007)

[15] L. Petrov, Y.Y. Kovalev, E.B. Fomalont, D. Gordon, AJ 136, 580 (2008)

[16] M.L. Lister, H.D. Aller, M.F. Aller, M.H. Cohen, D.C. Homan, M. Kadler, K.I. Kellermann, Y.Y. Kovalev, E. Ros, T. Savolainen et al., AJ 137, 3718 (2009)

[17] M.C. Shepherd (San Francisco: ASP, 1997), Vol. 125 of Astronomical Society of the Pacific Conference Series, p. 77

[18] I. Agudo (2009), Vol. 402 of Astronomical Society of the Pacific Conference Series, p. 330

[19] T. Savolainen, K. Wiik, E. Valtaoja, M. Tornikoski, A\&A 446, 71 (2006)

[20] A.P. Lobanov, J. Roland, A\&A 431, 831 (2005)

[21] U. Bach, T.P. Krichbaum, E. Ros, S. Britzen, W.W. Tian, A. Kraus, A. Witzel, J.A. Zensus, A\&A 433, 815 (2005)

[22] A.M. Stirling, T.V. Cawthorne, J.A. Stevens, S.G. Jorstad, A.P. Marscher, M.L. Lister, J.L. Gómez, P.S. Smith, I. Agudo, D.C. Gabuzda et al., MNRAS 341, 405 (2003)

[23] I. Marti-Vidal, J.M. Marcaide, A. Alberdi, A. Brunthaler, ArXiv e-prints, 1301.4782 (2013)

[24] S.G. Jorstad, A.P. Marscher, M.L. Lister, A.M. Stirling, T.V. Cawthorne, J.L. Gómez, W.K. Gear, AJ 127, 3115 (2004)

[25] I. Agudo, U. Bach, T.P. Krichbaum, A.P. Marscher, I. Gonidakis, P.J. Diamond, M. Perucho, W. Alef, D.A. Graham, A. Witzel et al., A\&A 476, L17 (2007)

[26] P.E. Hardee (2011), Vol. 275 of IAU Symposium, pp. 41-49

[27] N.R. Lomb, Ap\&SS 39, 447 (1976)

[28] J.D. Scargle, ApJ 263, 835 (1982)

[29] T. Hovatta, M.L. Lister, M.F. Aller, H.D. Aller, D.C. Homan, Y.Y. Kovalev, A.B. Pushkarev, T. Savolainen, AJ 144, 105 (2012)

[30] G.C. Duncan, P.A. Hughes, ApJ 436, L119 (1994)

[31] A. Rosen, P.A. Hughes, G.C. Duncan, P.E. Hardee, ApJ 516, 729 (1999) 
[32] A.P. Lobanov, J.A. Zensus, Science 294, 128 (2001)

[33] M. Perucho, Y.Y. Kovalev, A.P. Lobanov, P.E. Hardee, I. Agudo, ApJ 749, 55 (2012)

[34] R.T. Zavala, G.B. Taylor, ApJ 626, L73 (2005)

[35] K. Asada, M. Inoue, S. Kameno, H. Nagai, ApJ 675, 79 (2008)

[36] J.L. Gómez, M. Roca-Sogorb, I. Agudo, A.P. Marscher, S.G. Jorstad, ApJ 733, 11 (2011)

[37] R.C. Vermeulen, M.H. Cohen, ApJ 430, 467 (1994)

[38] M.L. Lister, A.P. Marscher, ApJ 476, 572 (1997)

[39] D.L. Meier, S. Koide, Y. Uchida, Science 291, 84 (2001)
[40] M. Sikora, M.C. Begelman, G.M. Madejski, J.P. Lasota, ApJ 625, 72 (2005)

[41] N. Vlahakis, A. Königl, ApJ 605, 656 (2004)

[42] D.C. Homan, M.L. Lister, AJ 131, 1262 (2006)

[43] A.A. Abdo, M. Ackermann, I. Agudo, M. Ajello, H.D. Aller, M.F. Aller, E. Angelakis, A.A. Arkharov, M. Axelsson, U. Bach et al., ApJ 716, 30 (2010)

[44] P. Natarajan, J.E. Pringle, ApJ 506, L97 (1998)

[45] A.T. Deller, W.F. Brisken, C.J. Phillips, J. Morgan, W. Alef, R. Cappallo, E. Middelberg, J. Romney, H. Rottmann, S.J. Tingay et al., PASP 123, 275 (2011) 